ABSTRACT submitted for: IEEE APWC 2018

Special Session titled: Emerging antenna technologies and wideband/multiband antennas

Organized by: Hisamatsu Nakano

\title{
Millimeter Wave Beam Steerable/Reconfigurable Liquid Metal Array Antenna
}

\author{
Khaled Yahya Alqurashi ${ }^{(1)}$, Carol Crean ${ }^{(2)}$, H.R.D. Filgueiras ${ }^{(3)}$, I. F. da Costa $^{(3)}$, Arismar \\ Cerqueira S. Jr. ${ }^{(3)}$, Pei Xiao ${ }^{(1)}$, Zhe Chen ${ }^{(4)}$, Hang Wong ${ }^{(4)}$, J. R. Kelly ${ }^{(1)}$ \\ (1) Institute for Communication Systems (ICS), University of Surrey, Guildford, UK; e-mail: \\ k.alqurashi@surrey.ac.uk \\ (2) Department of Chemistry, University of Surrey, Guildford, UK \\ (3) Laboratory WOCA, Inatel, Santa Rita do Sapucaí, Brazil \\ (4) The State Key Laboratory of Millimeter Waves, City University of Hong Kong, Hong Kong,
}

\section{Introduction:}

Users within mobile networks require ever increasing data rates. However, the frequency spectrum, reserved for mobile networks, is highly saturated. The millimeter wave spectrum, by contrast is relatively under utilised. Nonetheless, this area of the spectrum suffers from higher propagation losses, necessitating the use of highly directional antennas. To support mobility these antennas require beam steering capabilities. For several applications wide beam scanning capability is required. A valuable approach for increasing the beam scanning range is to use element factor plus array factor control [1]. Although several authors have presented designs based on this approach the lobe performance of those antennas is generally quite poor. In this paper we seek to address that issue. Moreover we employ liquid metal to dynamically reconfigure the direction of each element beam. There is a limited about of published work on the topic of using liquid metal to create a beam reconfigurable mm-wave antenna. A focal plane array is presented in [2]. The antenna incorporated a number of interconnected reservoirs. When a reservoir is filled by mercury, it acts as a patch antenna. The beam can be steered by filling and employing different combinations of patches. The antenna operates at $30.2 \mathrm{GHz}$ however it has a beam scanning range of only up to $\pm 30^{\circ}$ and utilises a hazardous material (mercury). In this paper a much safer liquid metal, based on gallium alloy, is used. The beam scanning approach proposed here also supports continuous beam steering over a much wider scan angle range than is possible with conventional techniques.

\section{Antenna Design}

Figure $1(\mathrm{a}, \mathrm{b})$ and $(\mathrm{c})$ show the radiating element and array antenna, respectively. The antenna is based on a design from the literature [3]. The radiating element consists of a pair of printed dipoles and two pairs of parasitic elements, which act as reflectors. The antenna is based on a $0.25 \mathrm{~mm}$-thick Rogers RT5880 substrate having a permittivity of 2.2 and a loss tangent of 0.0009 at $10 \mathrm{GHz}$. 


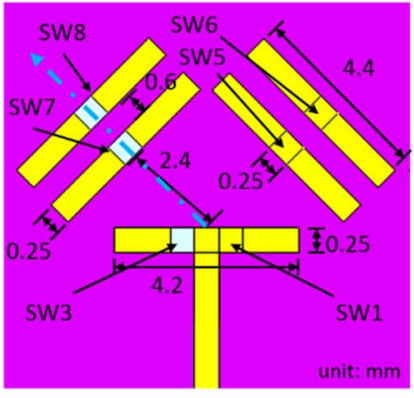

(a)

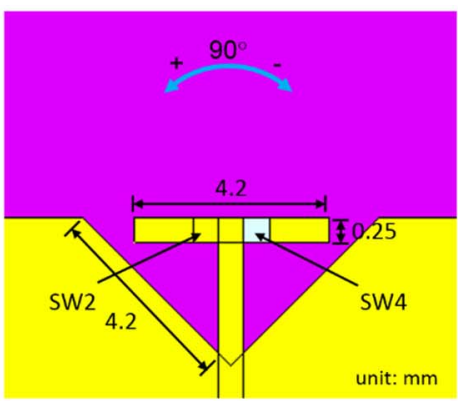

(b)
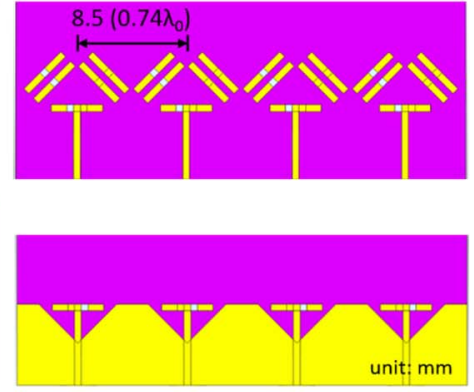

(c)

Figure 1: Antenna structure: (a) top view; (b) bottom view; (c) array top and bottom views.

Table 1 explains how to alter the main beam direction associated with the radiating element. Note that Figure $1(\mathrm{a}, \mathrm{b})$ presents the radiating element in state 1 and thus the main beam direction is as indicated by the dashed blue arrow. Each dipole arm and parasitic incorporates a single microwave switch. These switches are formed from liquid metal. We have employed a commercially available liquid metal known as EGaIn. This material has a conductivity of $3.4 \times 10^{6} \mathrm{~S} / \mathrm{m}$.

Table 1: Operating states.

\begin{tabular}{|c|c|c|}
\hline Operating State & Single Element Beam Direction & Active Switches \\
\hline 1 & $+34^{\circ}$ & SW1, SW2, SW5, SW6 \\
\hline 2 & $0^{\circ}$ (i.e. end fire) & SW1, SW2 or SW3, SW4 \\
\hline 3 & $-34^{\circ}$ & SW3, SW4, SW7, SW8 \\
\hline
\end{tabular}

\section{Simulation Results}

Figure 2 shows example of radiation patterns (realized gain) for the novel antenna in states 1 and 2 . The patterns are plotted at the operating frequency of the antenna $(26.2 \mathrm{GHz})$.

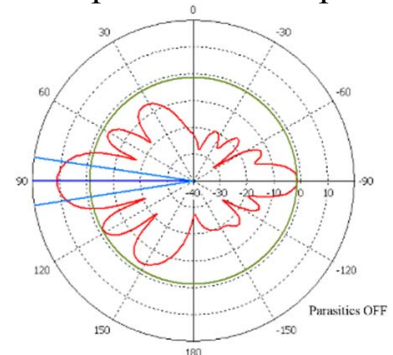

(a)

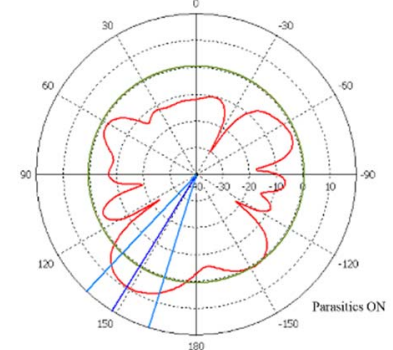

(c)

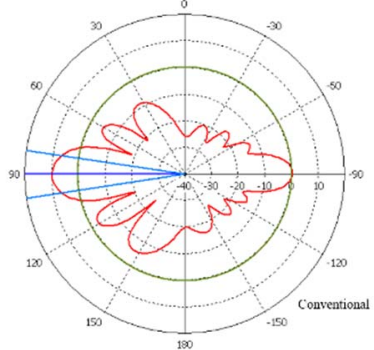

(b)

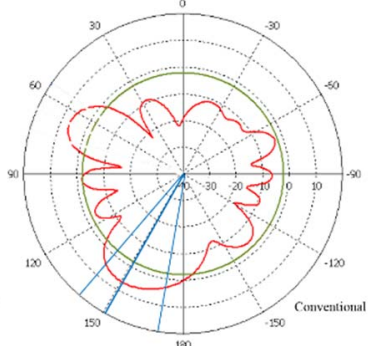

(d)

Figure 2: Radiation patterns for novel (a: state 2, c: state 1) and conventional antenna (b, d) when phase shift between consecutive elements of the array is set to: $(a, b) 0^{\circ}$, and (c, d) 237.5 . 
In order to obtain the optimum results from the novel array antenna we use state 2 , when scanning the beam between 0 and $\pm 15^{\circ}$. By using state 1 or 3 , as required we can then scan the beam out to $+58^{\circ}$ or $-58^{\circ}$, respectively. This approach ensures a lobe level of $8.8 \mathrm{~dB}$ or better throughout the scan range. The conventional phased array (i.e. the radiation patterns shown in Figure 2 ( $b$ and d) with all parasitics removed) has a scan range of only $\pm 18^{\circ}$.

\section{Conclusion}

This paper presents a mm-wave beam steerable phased array antenna incorporating EGaIn liquid metal switches. The antenna has a scan range of $\pm 58^{\circ}$ which yields a $\pm 40^{\circ}$ improvement in scan range over a conventional phased array antenna at a side lobe level of or better than $8.8 \mathrm{~dB}$.

\section{References}

[1] X. Ding; Y.-F. Cheng; et al., "A Wide-Angle Scanning Planar Phased Array with Pattern Reconfigurable Magnetic Current Element," IEEE Trans. Ant. and Prop., 2017, Vol.: 65, Issue: 3, pp. 1434-39.

[2] A. Gheethan, R. Guldiken, and G. Mumcu, "Microfluidic enabled beam scanning focal plane arrays," in 2013 IEEE Antennas and Propagation Society International Symposium (APSURSI), 2013, pp. 208-209.

[3] Z. Lu and X. Yang, "A novel planar beam steering antenna," in 2013 IEEE International Wireless Symposium (IWS), 2013, pp. 1-4. 